\title{
RAO-BLACKWELLIZED RESAMPLING PARTICLE FILTER FOR REAL-TIME PLAYER TRACKING IN SPORTS
}

\author{
Nicolai v. Hoyningen-Huene, Michael Beetz \\ Intelligent Autonomous Systems Group, Technische Universität München, Boltzmannstr. 3, D-85748 Garching, Germany \\ hoyninge,beetz@cs.tum.edu
}

Keywords: $\quad$ Multi-target tracking, Particle Filter, Rao-Blackwellization, Kalman Filter, Resampling

\begin{abstract}
Tracking multiple targets with similiar appearance is a common task in computer vision applications, especially in sports games. We propose a Rao-Blackwellized Resampling Particle Filter (RBRPF) as an implementable real-time continuation of a state-of-the-art multi-target tracking method. Target configurations are tracked by sampling associations and solving single-target tracking problems by Kalman filters. As an advantage of the new method the independence assumption between data associations is relaxed to increase the robustness in the sports domain. Smart resampling and memoization is introduced to equip the tracking method with real-time capabilities in the first place. The probabilistic framework allows for consideration of appearance models and the fusion of different sensors. We demonstrate its applicability to real world applications by tracking soccer players captured by multiple cameras through occlusions in real-time.
\end{abstract}

\section{INTRODUCTION}

Tracking multiple targets is needed in a lot of computer vision applications like surveillance or sports analysis. The sports domain provides a challenging testbed for concurrent tracking of multiple targets with similar appearance through frequent occlusions measured from different views. In team sports the complex coordination of movements of different players are crucial to the success of a squad. For automated analysis thereof the correct association of players to movements is equally important as the recognition of the movement itself.

To achieve an automatic extraction of athlete positions during sports games from video streams, beside camera estimation and player segmentation, a robust and fast multi-target tracking method is needed. In sports games the number of players is usually known and constant. In contrast the number of observations for each player obtained by measurements from sensors or segmentation for videos varies; it ranges from zero in case of occlusion and oversight to several measurements in case of hallucination and inaccuracy of the player extraction. Players usually differ by appearance from the field to help viewers and referees to follow the game easily, so the association of players of one team with their individual name is the bigger problem.

In this paper we propose a Rao-Blackwellized Resampling particle filter (RBRPF) for real-time tracking of multiple targets. Particles are represented as configurations of all players to result in tracking a mixture of Gaussians, where the multi-modality is caused by possible mix-ups of associations and the Gaussian refers to the uncertainty of dynamics. Sampling of new target configurations is reduced to sampling associations and Rao-Blackwellized by using the Kalman filter. Taking advantage of the fact that the number of probable associations for given player positions and measurements are usually low, the particle filter focuses on the most likely associations and can avoid unnecessary computations by smart resampling and memoization. The Bayesian framework allows the integration of kinematic and appearance models to determine the most probable player locations through occlusions.

Our contributions are the enhancements of a stateof-the-art theoretical multi target tracking method towards an implementable real-time algorithm that performs well in the demanding sports domain. We relax 
the independence assumption of single measurement associations to suit the original method to the application domain and achieve more robustness. Further we invent a smart resampling procedure that allows realtime in the first place and adapts to the complexity of the tracking problem. The proposed memoization of repeatedly computed results additionally improves efficiency.

The method is developed as part of the AsPoGAMO system (Beetz et al., 2006; Beetz et al., 2007), that aims to extract knowledge from broadcasted soccer games, and is evaluated by applying it to real soccer games, showing robust real-time performance over challenging sequences.

The remainder of this paper is organized as follows. We briefly talk about related work in the next section. In section 3 we derive the Rao-Blackwellized Resampling particle filter. Section 4 describes the experiments we conducted. We finish in section 5 with our conclusions.

\section{RELATED WORK}

Multiple-target tracking algorithms can be differentiated by their data association methods. Multiple hypothesis tracking (MHT) (Bar-Shalom et al., 2001) builds a pruned tree of all possible association sequences of each measurement with close targets by the Hungarian algorithm. The assumption of single associations and the use of Kalman filtering allow computation in polynomial time, but inhibit to handle multiple or merged associations. Khan, Balch \& Dellaert (2006) propose a real-time Rao-Blackwellized MCMC-based particle filter where associations are sampled by a Markov chain. The Markov chain allows also sampling of merged measurement assignments but demands computation time that reduces the number of particles. In their experiments real-time could only be provided for a small number of particles (less than 6) i.e. the tracker can cope with three parallel mix-ups of targets max. Interaction of targets are modeled as correlations between target positions which does not hold for many applications.

The Rao-Blackwellized particle filter approach by Särkkä et al. (2004; 2007) samples the associations directly and handles dependencies between them by data associations priors. The performance of the method was demonstrated only on synthetic simulations without statements about computation time. Our approach is an extension of this method to real world applications introducing smart resampling and memoization that leads to real-time tracking in the first place and relaxation of the association independence assumption.

Tracking of soccer players is classified by $\mathrm{Li}$ et al. (2005) in category and identity tracking. Category tracking extracts trajectories with team affiliation where in the other case each single player is traced with its identity. Barceló et al. (2005) and Figueroa et al. (2006) label the measurements by nearest neighbor assignment. In Gedikli et al. (2007) MHT was applied, but Particle filters constitute the mostly used method in the literature for category tracking (i.e. (Yang et al., 2005; A.Dearden \& Grau, 2006)). Du et al. (2006; 2007) aim on combining local particle filters to fuse measurements captured from different views. A MCMC method for team labelling is proposed by Liu et al. (2007) to link observations of soccer players over time.

Identity tracking is often performed in a second stage by consistent labelling of the trajectory graph generated by category tracking. Huang \& Hilton (2006) propose an assignment in batch mode by shortest path algorithm, Nillius et al. (2006) solve the association of the trajectory graph by Bayesian network inference, and Sullivan \& Carlsson (2006) combine trajectories of unoccluded players in a graph structure by clustering. Barceló et al. (2005) resolve collisions of nearest neighbor Kalman tracking by constraints in the trajectory graph. To the best of our knowledge no real-time identity tracking method for soccer player that allows multiple measurements and fuses different camera views was proposed in the literature yet.

\section{RAO-BLACKWELLIZED RESAMPLING PARTICLE FILTER}

A particle filter for complete player configurations constitutes the base of our algorithm. New particles are predicted by sampling associations of players with current measurements considering dependencies between them. Computation time is spend mostly on the highly probable configurations and on ambiguous associations by memoization of precomputed samples and probabilities. Sampling and weighting is done by using the Kalman filter for Rao-Blackwellization of the particle filter.

\subsection{Bayesian View of Tracking}

The problem of tracking is to recursively estimate a state $x_{k}$ knowing the evolution of the state sequence

$$
x_{k}=f_{k}\left(x_{k-1}, v_{k-1}\right)
$$


from measurements

$$
z_{k}=h_{k}\left(x_{k}, n_{k}\right)
$$

where $f_{k}$ is called system or motion model and $h_{k}$ is called measurement model, $v_{k-1}$ and $n_{k}$ denote the process and measurement noise, respectively. The tracked state $x_{k}$ is represented as the configuration of all player states

$x_{k}=\left\{x_{j, k}=\mathcal{N}\left(\left(\begin{array}{c}x \\ y \\ \dot{x} \\ \dot{y}\end{array}\right) ; m_{j, k}^{i}, V_{j, k}\right)\right\} j=1, \ldots, T$

where $x_{j, k}$ contains the position and velocity of player $j$ at time $k$. An individual target sample $x_{j, k}^{i}$ is assumed to be Gaussian with mean $m_{j, k}^{i}$ and corresponding covariance matrix $V_{j, k}^{i}$.

In a Bayesian framework, the problem of tracking can be formulated as one of estimating the posterior probability density function $p\left(x_{k} \mid z_{1: k}\right)$ for the state $x_{k}$ given a sequence of measurements $z_{1: k}$ up to time $k$.

\subsection{Particle Filtering}

In Sampling Importance Sampling (SIS) particle filtering, the posterior probability density function is approximated by a weighted sum of random samples $x_{k}^{i}$ also called particles (Arulampalam, Maskell, Gordon $\&$ Clapp, 2002). The weights are normalized such that $\sum_{i} w_{k}^{i}=1$ :

$$
p\left(x_{k} \mid z_{1: k}\right) \approx \sum_{i} w_{k}^{i} \delta\left(x_{k}-x_{k}^{i}\right) .
$$

We draw samples $x_{k}^{i}$ by importance sampling from a proposal $q($.$) called an importance density.$ Doucet (1998) showed that the optimal importance density function that minimizes the variance of the true weights conditioned on $x_{k-1}^{i}$ and $z_{k}$ is

$$
q_{\text {opt }}\left(x_{k} \mid x_{k-1}^{i}, z_{k}\right)=\frac{p\left(z_{k} \mid x_{k}, x_{k-1}^{i}\right) p\left(x_{k} \mid x_{k-1}^{i}\right)}{p\left(z_{k} \mid x_{k-1}^{i}\right)} .
$$

In our case the importance density is the probability distribution of data associations, while the actual sample is deduced from an association by the use of Kalman filtering.

\subsection{Sampling new Configurations}

For known associations between measurements $z_{k}$ and players of the sample $x_{k-1}^{i}$ the new sampled configuration is Gaussian and can be evaluated analytically as an optimal fusion between measurements and predicted player positions. The Kalman filter provides the method to find the Gaussian that equals both probabilities in the numerator of equation 5 and thus maximizes their product. The sampling problem reduces therefor to sample associations between measurements and the predicted player configuration and solving multiple single target tracking problems by Kalman filtering. The analytical sampling part forms the Rao-Blackwellization of the particle filter. To supply an optimal solution the Kalman filter assumes state and measurement noise to be zero-mean, white Gaussian and the measurement as well as the motion model to be linear. If the last assumption does not hold an extended or unscented Kalman filter could be applied for a suboptimal solution. Following this approach the posterior probability density function of configurations form a mixture of Gaussians, where the multi-modality originates from ambiguities in the associations.

\subsubsection{Predicting by System Model}

We can sample from $p\left(x_{k}^{\prime} \mid x_{k-1}^{i}\right)$ analytically by the Kalman prediction step according to the system dynamics of eq. 1. Each player state is predicted independently using the discretized Wiener velocity model $A_{\Delta t}$ (Bar-Shalom, Li \& Kirubarajan, 2001) for time difference $\Delta t$ between $k-1$ and $k$ as a linear motion model:

$m_{j, k}^{\prime}=\left(\begin{array}{c}x_{j, k}^{\prime} \\ y_{j, k}^{\prime} \\ \dot{x}_{j, k}^{\prime} \\ \dot{y}_{j, k}^{\prime}\end{array}\right)=\left(\begin{array}{cccc}1 & 0 & \Delta t & 0 \\ 0 & 1 & 0 & \Delta t \\ 0 & 0 & 1 & 0 \\ 0 & 0 & 0 & 1\end{array}\right)\left(\begin{array}{c}x_{j, k-1}^{i} \\ y_{j, k-1}^{i} \\ \dot{x}_{j, k-1}^{i} \\ \dot{y}_{j, k-1}^{l}\end{array}\right)$

The covariance matrix evolves to

$$
V_{k}^{\prime}=A_{\Delta t} V_{k-1} A_{\Delta t}^{T}+\left(\begin{array}{cccc}
\frac{\Delta t^{3}}{3} & 0 & \frac{\Delta t^{2}}{2} & 0 \\
0 & \frac{\Delta t^{3}}{3} & 0 & \frac{\Delta t^{2}}{2} \\
\frac{\Delta t^{2}}{2} & 0 & \Delta t & 0 \\
0 & \frac{\Delta t^{2}}{2} & 0 & \Delta t
\end{array}\right) \tilde{q}(7)
$$

with power spectral density $\tilde{q}$ as a constant factor.

\subsubsection{Sampling Associations}

We introduce associations

$$
J_{k}:\left\{1, \ldots,\left|z_{k}\right|\right\} \rightarrow \wp(\{1, \ldots, T\})
$$

as mappings from all measurements at time $k$ to a (possibly empty) subset of all targets. We denote $\hat{J}_{k}$ as the inverse mapping from targets to their assigned observations for convenience. The space of data associations equals the finite and discrete set of all possible associations of measurements to targets 
containing $2^{\left|z_{k}\right| \times T}$ elements. If we restrict the data associations $J_{k}$ to assign a measurement to one target max, the number of possible associations reduce to $(T+1)^{\left|z_{k}\right|}$. We can further reduce this number to $\sum_{i=0}^{\min \left(T,\left|z_{k}\right|\right)}\left(\begin{array}{c}\min \left(T,\left|z_{k}\right|\right) \\ i\end{array}\right) \max \left(T,\left|z_{k}\right|\right)^{\min \left(T,\left|z_{k}\right|\right)-i}$ if we prohibit multiple measurements per target, also called exclusion principle (MacCormick \& Blake, 1999). Enumerating this set and solving each single target tracking problem is still intractable even for a small number of targets and measurements. Fortunately only a few associations have high probability, but to sample them efficiently, we have to assume the associations for single measurements to be independently or the dependency between them to be determined fast.

Individual independent associations If we look at sampling an individual association for measurement $z \in z_{k}$, we can enumerate all possible assignments easily as $z$ can be clutter viz. a false alarm or assigned to one of the players. Thus the importance distribution $\pi(z)$ for an association of a specific measurement $z$ can be evaluated by normalizing the probabilities $\hat{\pi}(z)$ for each possible association.

Clutter measurements are assumed to be independent from player positions and uniformly distributed in the measurement space with volume $M$

$$
\hat{\pi}_{\varnothing}(z)=p\left(J_{k}(z)=\varnothing \mid z_{k}\right) \sim \frac{1}{M} .
$$

The probability for a data association between target $t$ and an observation $z$ is up to a constant factor:

$$
\begin{aligned}
\hat{\pi}_{t}(z) & =p\left(t \in J_{k}(z) \mid z_{k}, x_{k}^{\prime}\right) \\
& \sim p_{a p p}\left(t \in J_{k}(z)\right) N\left(z ; H_{z} m_{t, k}^{\prime}, H_{z}^{T} V_{t, k}^{\prime} H_{z}+R_{z}\right)
\end{aligned}
$$

with measurement model $H_{z}=\left(\begin{array}{cccc}1 & 0 & 0 & 0 \\ 0 & 1 & 0 & 0\end{array}\right)$ and $R_{z}$ as measurement noise covariance. $p_{\text {app }}\left(t \in J_{k}(z)\right)$ denotes the propability of an association based on the appearance model only, which is independent from player and measurement positions. The Gaussian in the second part refers to the probability of the association by the kinematic model. We included the appearance model in difference to (Särkkä et al., 2007) to allow a realistic influence of additional information from segmentation beside spatial data only.

Importance density Utilizing the independence of single associations the importance density for a sampled state $x_{k}^{j}$ can be computed as a product over probabilities of assignments for each single measurement
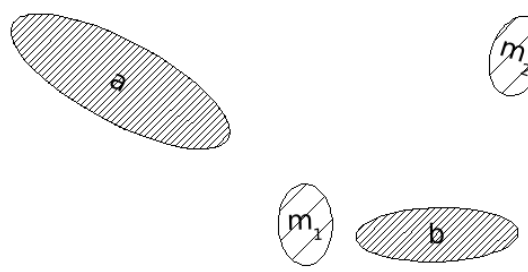

Figure 1: Association of $a$ and $m_{1}$ increases the probability of $b$ and $m_{2}$ being associated.

that are given by the normalized importance distribution $\pi$ of equations 9 and 10 .

$$
q\left(x_{k} \mid x_{k-1}^{i}, z_{k}\right)=\prod_{z_{k}} \pi
$$

Relaxation of Independence In the underlying method by Särkkä et al., the measurements are processed one at a time in sequential fashion based on the independence assumption of associations of individual measurements. This assumption does not always hold, the order of associations often do matter. This can be best exemplified by figure 1 assuming that measurements can be assigned to one target at max: If target $a$ is assigned to measurement $m_{1}$, the probability of the association of $m_{2}$ and target $b$ increases.

Särkkä et al. did not consider this problem at all but proposed the use of an data association prior. We follow this solution instead of establishing an additional Markov Chain as proposed by Khan et al. (2006) in favor of computational efficiency but change the procedure slightly to improve robustness against violation of the mentioned assumption. To generate new particles $x_{k}^{j}$ including the whole player configuration, we repeatedly sample an ordering on the measurements of one sweep uniformly at random, reducing the relevance of the ordering and the induced dependencies on the tracking result. With the randomly sampled ordering we draw an association for each measurement with the normalized importance distribution $\pi(z)$ one at a time. If a target was associated, it is excluded from further associations with the single detection probability $p_{s d}$ and the importance distribution is renormalized. If the mentioned exclusion principle holds i.e. targets can be assigned to one measurement at max, $p_{s d}$ should be set to one.

Determination of State from Associations For sampled associations $J_{k}$ the predicted player positions $x_{k}^{\prime}$ can be updated individually by Kalman update with their observations

$x_{j, k}^{i}=x_{j, k}^{\prime}+V_{j, k}^{\prime} H^{T}\left(H V_{k}^{\prime} H^{T}+R\right)^{-1}\left(\hat{J}_{k}(j)-H x_{j, k}^{\prime}\right)$, 
with $H$ denoting the linear measurement model (2) as $H_{z}$ stacked $\left|\hat{J}_{k}(j)\right|$ times and $R$ as diagonal matrix of measurement covariances of observations $\hat{J}_{k}(j)$.

\subsection{Weighting}

For a good performance of the particle filter the computation of the weights of each sampled state is crucial. To approximate $p\left(x_{k} \mid z_{1: k}\right)$ correctly the weights $w_{k}^{i}$ have to be defined recursively as

$$
w_{k}^{i} \propto w_{k-1}^{i} \frac{p\left(z_{k} \mid x_{k}^{i}\right) p\left(x_{k}^{i} \mid x_{k-1}^{i}\right)}{q\left(x_{k}^{i} \mid x_{k-1}^{i}, z_{k}\right)} .
$$

The denominator was already computed in the sampling phase and was depicted in equation 11 . The likelihood of the measurements given the sampled state $x_{k}^{i}$ with known associations and the likelihood of $x_{k}^{i}$ given the former state $x_{k-1}^{i}$ and the dynamics can be computed for each player and measurement separately. The measurement likelihood can be computed analogously to eq. 10 but substituting $x_{k}^{\prime}$ by $x_{k}^{i}$ and $V_{k}^{\prime}$ by $V_{k}^{i}$, respectively:

$$
p\left(z_{k} \mid x_{k}^{i}\right)=\prod_{z \notin \hat{J}_{k}^{i}} \hat{\pi}_{\varnothing}(z) \prod_{j} p\left(\hat{J}_{k}^{i}\left(x_{j, k}^{i}\right) \mid x_{j, k}^{i}\right) .
$$

The likelihood of the new sample according to the motion model can be computed by reusing the already predicted state $x_{k}^{\prime}$ of eq. 6

$$
p\left(x_{k}^{i} \mid x_{k-1}^{i}\right)=\prod_{j} N\left(m_{j, k}^{i} ; m_{j, k}^{\prime}, V_{j, k}^{i}+V_{j, k}^{\prime}\right) .
$$

\subsection{Resampling}

SIS particle filters suffer from the so called degeneracy phenomenon, where only a small amount of all particles have not negligible weights. This implies that most of the computation time will be spent on particles that contribute only marginally to the approximation of the posterior probability density function of equation 4 . To reduce the degeneracy problem resampling has been proposed to eliminate particles with small weights and clone the others according to their weights. We include the resampling step by sampling $w_{k-1}^{i} \times N_{\max }$ associations for particle $x_{k-1}^{i}$. Particles with larger weights will therefor allocate more particles in the next time step, while particles with small weights are dropped.

Sampling several times from the same particle the number of distinct sampled particles will approach the number of ambiguities in the associations because a specific assignment leads to the same sampled configuration. Due to their discreteness there are usually only a small number of distinct probable associations.
This allows a chance for noticeable improvement in computation time by smart memoization. Caching and testing sampled associations for equality can save computation time considering not only the update to generate a new state of equation 12 but also the prediction in the next particle filtering step in equation 6.

After resampling the weights are usually reset to $w_{k}=1 / N_{\max }$ to reflect the equal probability of all particles. In our case we count the times $n_{J_{k}}$ the same association $J_{k}$ was sampled for a specific particle and provide only one single particle for the next filtering step having the weight set to $w_{k}=n_{J_{k}} / N_{\max }$. Then the weights are recursively updated as in equation 13 and normalized at the end of the filtering step. The actual number of particles can therefor vary between 1 and $N_{\text {max }}$ using more particles in situations with high association ambiguities. This smart resampling reduces the computation time and allows real time in the first place.

\subsection{Estimate of the state}

An estimate of the player positions at time $k$ i.e. of the state $x_{k}$ can be found by either selecting the particle with maximum weight or by clustering the particles and taking the weighted mean of the most probable cluster. Calculating the weighted mean of all particles should not be considered here because it can lead to the so called ghost phenomenon for multi-modal distributions i.e. it leads to a state estimated as the mean of two modes that is known to be wrong.

\subsection{Implementation}

The complete algorithm is depicted in figure 2 following the derivation of the former section. The individual importance distributions $\pi$ as well as $\hat{\pi}$ and the Kalman prediction and updates are cached for reuse in the next sampling iteration to improve efficiency. The importance distribution, all probabilities and weights are calculated in log-space to avoid numerical problems.

\section{EXPERIMENTAL RESULTS}

The proposed tracking method is evaluated as part of the ASPOGAMO system (Beetz et al., 2006; Beetz et al., 2007), that aims to extract knowledge from broadcasted soccer games. ASPOGAMO is able to track multiple dynamic pan-tilt-zoom cameras and segment the soccer players and referee by a combination of 


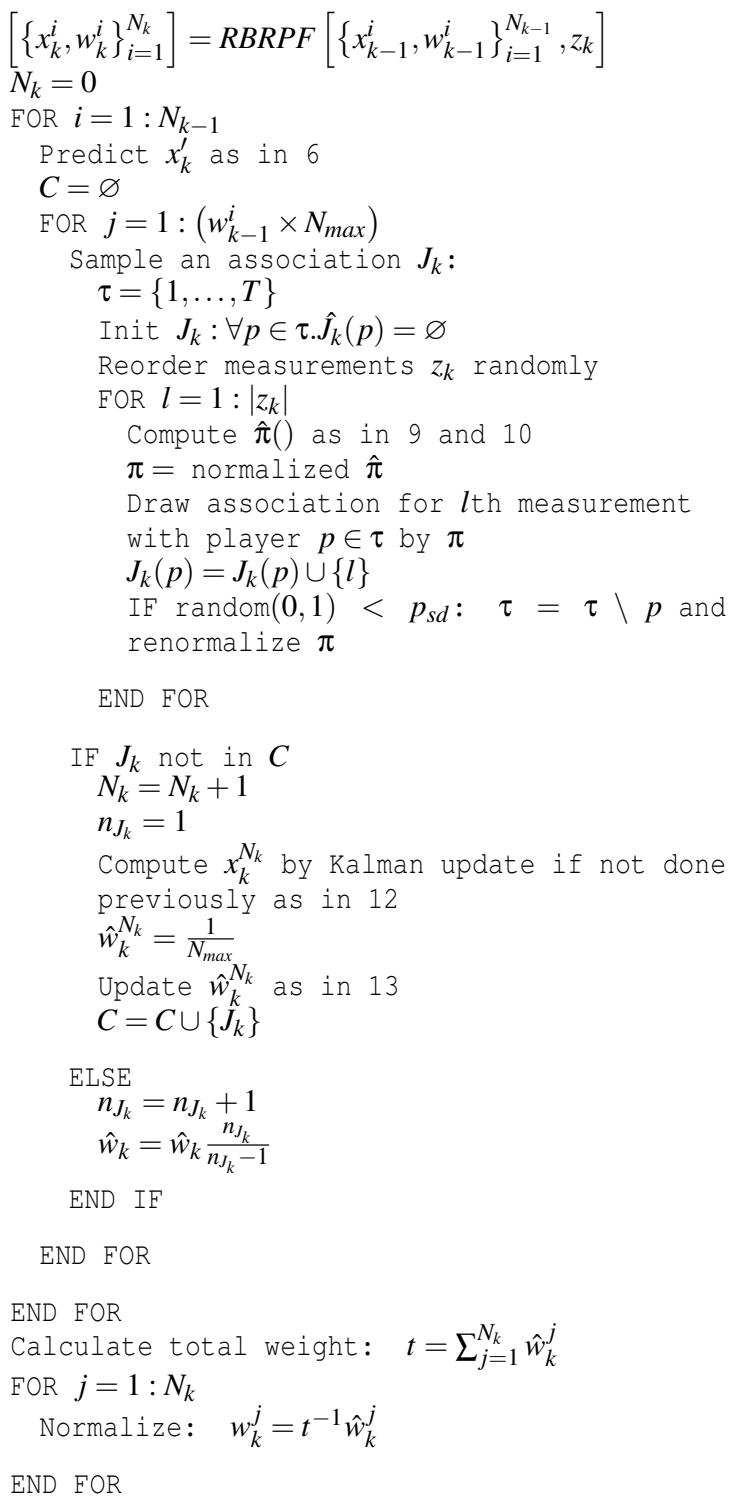

Figure 2: Algorithm for one iteration of the proposed RaoBlackwellized Resampling particle filter.

variance filter and color templates. Segmentation influences the tracking process as the Kalman filters smooth assigned measurements, quality evaluation of the used method can be found in (Beetz et al., 2007). However segmentation by background subtraction for static cameras is usually of higher quality. Digital videos captured by two dynamic cameras with a frame rate of $25 \mathrm{~Hz}$ provide the basic raw material. Tracking results in both camera perspectives are depicted in figures 3 and 4 and are presented quantitatively in table 2. The extracted players spatial measurements of each camera are fused by the proposed tracking al- gorithm as different measurement sweeps with same time stamps.

Player positions have been measured in meters and were initialized manually in the image with covariance $V_{0}=2 I_{4}$, initial velocity was set to zero. The factor for the kinematic process noise $\tilde{q}=0.0008$ is derived from maximal human speed. The probability of multiple observations for the same target was obtained experimentally to $p_{s d}=0.92$. A confusion matrix between different categories was used as a simple appearance model $p_{a p p}$ and is depicted in table 4. The measurement space is determined by the number of pixels in each camera frame and evaluates to $M=720 \times 576$. We used $N_{\max }=50$ particles to track all of the 22 players and the referee.

There is no ground truth for broadcasted soccer games because players can be tracked only visually and camera parameters are unknown. We abandon to present a spatial error as this is influenced mainly by camera estimation and segmentation. Instead we tried to find a error measure that is related with the number of false associations. A failure was counted when the projected player position differed from the real player in the image by more than 10 pixels for longer than 3 frames. In this case the tracker was reset in the failed player positions and run again on the rest of the sequence. We tracked both camera views separately and also ran the same sequence fusing the measurements of both perspectives. Because the broadcasted highangle camera shows only a part of the field and is panning and zooming fast, in average only 9.9 players are visible (with standard deviation of 3.2). We splitted the number of failures into association errors and assigning emerging players (second number) to be comparable with the other results. The second row of table 2 shows the number of frames that were tracked in the according experiment. The computation time was taken for one update step, where all experiments have been conducted on a $2.2 \mathrm{GHz}$ Dual-core PC. We think the actual needed time is more significant than the theoretical complexity of the algorithm since the input data do not scale but stay in fixed boundaries (number of players is 22, number of measurements usually lower than 200). The last row depicts the average number of particles and the corresponding standard deviation. Table 2 clearly evidences the real-time tracking ability of the proposed method with low failure rate for single cameras. Fusion of different cameras reduces the occurrence of occlusions and therewith failure rate and number of particles even further.

The fourth experiment states a challenging sequence including several fouls and header duels where kinematic and appearance model have often been to weak to differentiate between players causing 


\begin{tabular}{c|ccc} 
& Italy & France & Referee \\
\hline & & & \\
Italy & 0.6 & 0.1 & 0.3 \\
France & 0.1 & 0.8 & 0.1 \\
Referee & 0.3 & 0.1 & 0.6
\end{tabular}

Table 1: Confusion matrix between different categories.

\begin{tabular}{ccccc} 
Game & Frames & Fail & Time $(\mathrm{ms})$ & Particles \\
\hline Tactical & 1262 & 13 & $23.3 \pm 4$ & $43.5 \pm 10$ \\
Highangle & 1262 & $7+54$ & $8.5 \pm 5$ & $12.1 \pm 12$ \\
Fused & 1262 & 11 & $30.2 \pm 20$ & $33.3 \pm 16$ \\
Fused II & 3202 & 98 & $33.4 \pm 18$ & $34.1 \pm 14$
\end{tabular}

Table 2: Tracking performance on the final of the world cup 2006.

a higher number of failures. The amount of measurements (lower for the highangle view) correlates obviously with the number of particles and the computation time demonstrating the adaptiveness of the proposed method to the complexity of the tracking problem. Also we observed assignment errors if segmentation could not extract a specific player for longer than 20 frames (e.g. fouled player on the ground).

We also implemented the method as proposed by Khan et al. (2006) and tested it on the World Cup final. We encountered problems of two kind: low variance in sparse particles and misleading interaction handling. The real-time requirement allowed only a small number of particles (6 in our case) which had a low variance because the Markov chain converged to very similar associations. This misled the tracker to remember the most probable configuration only which often did not equal the true positions. Interactions are handled by dependencies in the positions via symmetric entries in the configuration covariance matrix. This modeling is inappropriate for interacting soccer players, where e.g. the player on the ball shows contrary motion to his competitor. Both drawbacks resulted in poor tracking performance for the inspected soccer game sequences.

\section{CONCLUSIONS}

In this article we have proposed a real-time multiple target tracking method based on Rao-Blackwellized Resampling particle filtering for tracking soccer player identities. We presented the necessary extensions of an so far only theoretically evaluated stateof-the-art multi-target tracking method to handle real

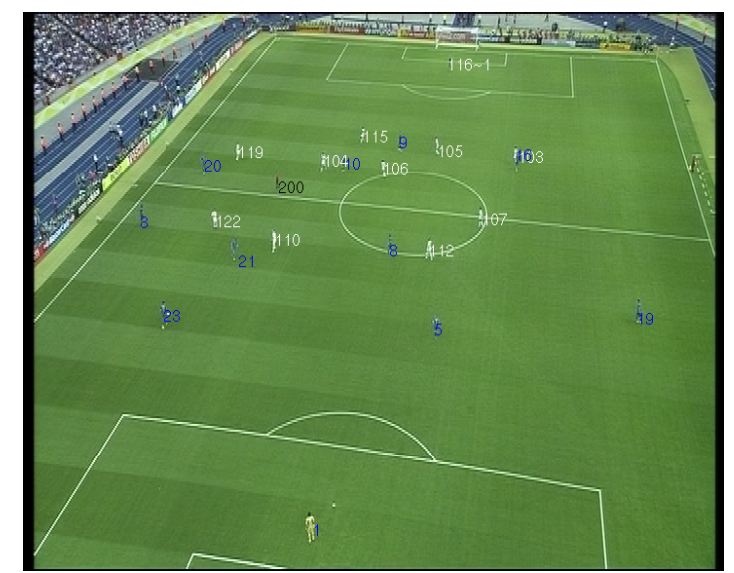

Figure 3: Tactical camera view of the World Cup final 2006.

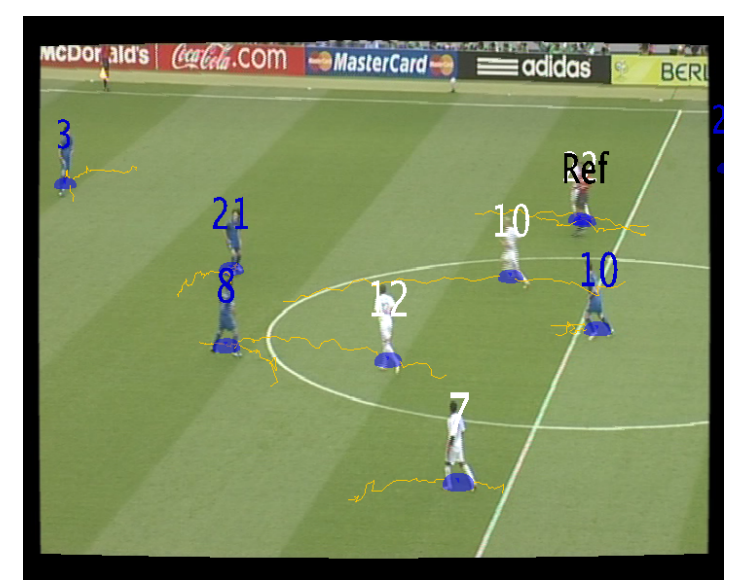

Figure 4: Identity tracking of soccer players in the broadcasted highangle camera view of the World Cup final 2006.

tracking problems being as challenging as in the sports domain. The first extension comprises the processing of measurements of one sweep instead of one at a time to relax the independence assumption of associations. Secondly, smart resampling and memoization was introduced to equip the tracking method with real-time capabilities. Experimental results demonstrate robustness and real-time performance of the developed method in challenging soccer game sequences including increased achievements by fusion of measurements from different cameras. A comparison with another recent multi-target tracking method explains the supremacy of our approach for the soccer domain. For future research we plan to examine more complex appearance models for automatic reinitialization of the identities especially regarding broadcasted single view sports videos. 


\section{ACKNOWLEDGEMENTS}

This work was partially funded by the German Research Foundation DFG.

\section{References}

A.Dearden, Y. \& Grau, O. (2006). Tracking football player movment from a single moving camera using particle filters. In European Conf. on Visual Media Production (CVMP 2006).

Arulampalam, M. S., Maskell, S., Gordon, N., \& Clapp, T. (2002). A tutorial on particle filters for online nonlinear/non-gaussian bayesian tracking. IEEE Trans. on Signal Processing, 50(2).

Bar-Shalom, Y., Li, X.-R., \& Kirubarajan, T. (2001). Estimation with Applications to Tracking and Navigation. Wiley Interscience.

Barceló, L., Binefa, X., \& Kender, J. R. (2005). Robust methods and representations for soccer player tracking and collision resolution. In Proc. of Intl. Conf. on Image and Video Retrieval (CIVR 2005), (pp. 237246).

Beetz, M., Bandouch, J., Gedikli, S., von HoyningenHuene, N., Kirchlechner, B., \& Maldonado, A. (2006). Camera-based observation of football games for analyzing multi-agent activities. In Proc. of Intl. Joint Conf. on Autonomous Agents and Multiagent Systems (AAMAS).

Beetz, M., Gedikli, S., Bandouch, J., Kirchlechner, B., von Hoyningen-Huene, N., \& Perzylo, A. (2007). Visually tracking football games based on tv broadcasts. In Proc. of Intl. Joint Conf. on Artificial Intelligence (IJCAI).

Doucet, A. (1998). On sequential Monte Carlo methods for Bayesian filtering. Technical report, Dept. End., Univ. Cambridge, UK.

Du, W., Hayet, J.-B., Piater, J., \& Verly, J. (2006). Collaborative multi-camera tracking of athletes in team sports. In Workshop on Computer Vision Based Analysis in Sport Environments (CVBASE), (pp. 2-13).

Du, W. \& Piater, J. H. (2007). Multi-camera People Tracking by Collaborative Particle Filters and Principal Axis-Based Integration. In Asian Conference on Computer Vision, number 4843 in LNCS, (pp. 365-374). Springer.

Figueroa, P. J., Leite, N. J., \& Barros, R. M. L. (2006). Tracking soccer players aiming their kinematical motion analysis. Computer Vision and Image Understanding, 101(2), 122-135.

Gedikli, S., Bandouch, J., von Hoyningen-Huene, N., Kirchlechner, B., \& Beetz, M. (2007). An Adaptive Vision System for Tracking Soccer Players from Variable Camera Settings. In Proc. of Intl. Conf. on Computer Vision Systems (ICVS).

Huang, P. \& Hilton, A. (2006). Football player tracking for video annotation. In European Conf. on Visual Media Production.
Khan, Z., Balch, T., \& Dellaert, F. (2006). Mcmc data association and sparse factorization updating for real time multitarget tracking with merged and multiple measurements. IEEE Trans. on Pattern Analysis and Machine Intelligence, 28(12), 1960-1972.

Li, Y., Dore, A., \& Orwell, J. (2005). Evaluating the performance of systems for tracking football players and ball. In IEEE Intl. Conf. on Advanced Video and Signal Based Surveillance.

Liu, J., Tong, X., Li, W., Wang, T., Zhang, Y., Wang, H., Yang, B., Sun, L., \& Yang, S. (2007). Automatic player detection, labeling and tracking in broadcast soccer video. In British Machine Vision Conference.

MacCormick, J. \& Blake, A. (1999). A probabilistic exclusion principle for tracking multiple objects. In Proc. of Intl. Conf. on Computer Vision (ICCV), (pp. 572578).

Nillius, P., Sullivan, J., \& Carlsson, S. (2006). Multi-target tracking - linking identities using bayesian network inference. In Proc. of Computer Vision and Pattern Recognition, (pp. 2187-2194).

Särkkä, S., Vehtari, A., \& Lampinen, J. (2004). Raoblackwellized monte carlo data association for multiple target tracking. In Proc. of Intl Conf. on Information Fusion, volume 7, Stockholm.

Särkkä, S., Vehtari, A., \& Lampinen, J. (2007). Raoblackwellized particle filter for multiple target tracking. Information Fusion Journal, 8(1), 2-15.

Sullivan, J. \& Carlsson, S. (2006). Tracking and labelling of interacting multiple targets. In Proc. of European Conf. on Computer Vision, (pp. 619-632).

Yang, C., Duraiswami, R., \& Davis, L. (2005). Fast multiple object tracking via a hierarchical particle filter. In Proc. of Intl. Conf. on Computer Vision, volume 1, (pp. 212-219). 